\section{Anesthetic management of pulmonary surgery in newborns and infants}

\author{
Marco Caruselli, ${ }^{1}$ Daniele Galvagni, ${ }^{1}$ \\ Julia Boubnova,2 Fabrice Michel1,3 \\ ${ }^{1}$ Anesthesia and Intensive Care Unit, La \\ Timone Children's Hospital, AP-HM, \\ Marseille; 2Diaphragmatic Hernias \\ Reference Center, Pediatric Visceral \\ Surgery Unit, La Timone Children's \\ Hospital, AP-HM, Marseille; ${ }^{3}$ AMU \\ UMR ADES 7268, Mediterranean \\ Ethical Space, Timone Adults, Marseille, \\ France
}

\begin{abstract}
The main congenital pulmonary airways malformations in newborns and infants requiring surgery are cystic adenoid malformation, congenital lobar emphysema and bronchogenic cyst. The surgical treatment preferably via thoracoscopy is recommended within the first year of life to avoid the risk of pneumopathy. A monopulmonary ventilation is then required by the surgeon to operate the diseased lung. The anesthetic management of intraoperative mono-pulmonary ventilation in newborns and infants is always challenging for the anesthesiologist. The main objective of this study was to describe anesthetic protocol for thoracoscopy and variations of monitored parameters during a mono-pulmonary ventilation procedure in newborns and infants.
\end{abstract}

\section{Introduction}

The main congenital pulmonary airways malformations (CPAM) in newborns and infants requiring surgery are cystic adenoid malformation, congenital lobar emphysema and bronchogenic cyst.

Cystic adenomatoid malformation is characterized by multiple cysts made of abnormal lung tissue secondary to an embryologic development defect. Newborns may develop a respiratory distress at birth caused by pulmonary hypoplasia and pulmonary arterial hypertension. In most cases, patients are not symptomatic but are often affected by pulmonary infections.

Congenital lobar emphysema is characterized by a hyperexpansion of a lobe of the lung causing the compression of pulmonary structures and mediastinum. It is thought to be the consequence of a bronchial cartilage development abnormality (bronchomalacia, bronchial stenosis or atresia). The severity of symptoms depends on the presence of air trapping phenomenon as well as the pace of emphysema evolution. This condition can become symptomatic from birth or within the first 6 months of life. Bronchogenic cyst is a congenital mucusproducing cyst secondary to an alveolar or pleural tissue abnormality. The presence of the cyst causes an indirect compression of airway or esophagus. It is also sometimes accompanied by abundant bronchial secretions that can lead to frequent pneumonia. ${ }^{1-3}$

The surgical treatment preferably via thoracoscopy is recommended within the first year of life to avoid the risk of pneumopathy. A mono-pulmonary ventilation is then required by the surgeon to operate the diseased lung. $4-6$

The anesthetic management of intraoperative mono-pulmonary ventilation in newborns and infants is always challenging for the anesthesiologist. It requires an extensive knowledge of the pediatric respiratory physiology and a considerable experience in pediatric anesthesia. While several articles have reported surgical techniques for thoracoscopy, anesthetic characteristics including clinical effect, tolerance, and management are poorly documented. . $^{-12}$ The aim of this retrospective study was to describe anesthesia practice in our institution and to compare the two techniques for the mono-pulmonary ventilation in newborns and infants.

\section{Materials and Methods}

We retrospectively analyzed charts of newborns and infants who underwent lung surgery for congenital malformation pulmonary diseases between January 1st 2013 and December 31th 2019 in our university hospital. The study was approved by the local Institutional Ethics Committee. The inclusion criteria of the patients were: i) age less than 24 months; ii) weight less than $15 \mathrm{~kg}$; iii) pulmonary surgery.

Data were extracted from patients' charts. The following variable were collected: cause of surgery, age at the time of the surgery, comorbidities, length of anesthetic management (from the admission in the operative room to the installation in lateral decubitus), length of surgery, complications of anesthesia and of surgery, unit where child was hospitalized after postoperative care unit, postoperative complications and length of stay. We analyzed the anesthetic protocol including monitored parameters, drugs used for
Correspondence: Marco Caruselli,Anaesthesia and Intensive Care Unit, Timone Children's Hospital, AP-HM, 264 Rue Saint-Pierre, 13385 Marseille, France.

Tel: +33.0491384944

E-mail: m.caruselli@gmail.com

Key words: congenital pulmonary airways malformations, newborn, infants, thoracic surgery, anesthesia.

Contributions: MC elaborated the layout of the article, analyzed the problems of monopulmonary ventilation in the newborn and infants and has written the article; DG participated in the writing of the article and in the statistical analysis; JB participated in the analysis of anesthesia problems compared to thoracic surgery techniques; FM participated in the drafting of the article and in the analysis of anesthesiological problems.

Conflict of interest: The authors declare no potential conflict of interest.

Funding: None.

Availability of data and materials: All data and materials are available upon request by the corresponding author

Informed consent: Not required.

Received for publication: 21 April 2020.

Revision received: 23 June 2020

Accepted for publication: 24 June 2020.

This work is licensed under a Creative Commons Attribution NonCommercial 4.0 License (CC BY-NC 4.0).

(C) Copyright: the Author(s), 2020

Licensee PAGEPress, Italy

Pediatric Reports 2020; 12:8595

doi:10.4081/pr.2020.8595

induction and maintenance, variation of respiratory parameters $(\mathrm{SpO} 2$, and $\mathrm{ETCO} 2)$ and of hemodynamic parameters (heart rate, arterial pressure).

\section{Statistics}

The general characteristics of the patients were expressed as means $( \pm \mathrm{SD}$ standard deviation) for quantitative variables and as frequencies (n, \%) for qualitative and discrete variables. Continuous data were evaluated using the student $t$-test, and other dichotomous data with Fisher's exact test. $P$ values $=0.05$ was used as cut off for statistical significance.

\section{Anesthetic protocol}

At the admission to the operative room 
all patients were monitored by electrocardiogram (ECG), pulse oximetry (SpO2), and non-invasive blood pressure.

The induction of anesthesia was carried out by inhaled $6 \%$ sevoflurane and $80 \%$ oxygen. Opioid (fentanyl 2,5 $\mathrm{mcg} / \mathrm{kg}$ or sufentanil $0,2 \mathrm{mcg} / \mathrm{kg}$ ) and muscle relaxant (atracurium $0,5 \mathrm{mg} / \mathrm{kg}$ or cis-atracurium 0,2 $\mathrm{mg} / \mathrm{kg}$ ) were administered after placement of a peripheral venous catheter. As soon as possible an oro-tracheal intubation was performed and mechanical bi-pulmonary ventilation was begun with a tidal volume of $7 \mathrm{ml} / \mathrm{kg}$. Continuous monitoring of expired $\mathrm{CO} 2$ was initiated (ETCO2) and an arterial catheter for invasive blood pressure monitoring was positioned in the radial or femoral artery. Exclusion of the operated lung and mono-pulmonary ventilation were obtained before placing the patient in lateral decubitus position. Two techniques were used: i) either the use of a $5 \mathrm{Fr}$ bronchial blocker positioned in the main bronchus of the operated lung; ii) or a selective intubation in the bronchus of non-operated lung. In the second case before proceeding to selective intubation the measurement of the endotracheal tube at the level of the dental arch was noted and used for the postoperative placement of the tube. All these maneuvers were carried out under fibroscopic control and thoracic auscultation confirmed the pulmonary unilateral ventilation. Just before selective intubation, tidal volume was set to $4-5 \mathrm{ml} / \mathrm{kg}$ with expiratory pressure to 5 $\mathrm{cmH} 2 \mathrm{O}$. Respiratory rate set according to the ETCO2 data. Anesthesia was maintained by MAC 1 of sevoflurane and opioids reinjections. At the end of the operation, before the chest was closed, bi-pulmonary ventilation was re-established to re-expand the lung excluded during surgery if necessary with manual pulmonary recruitment maneuvers.

In the case of patients with a bronchial blocker, the blocker balloon was simply deflated. In the case of selectively intubated patients the endotracheal tube was withdrawn up to the centimeters corresponding to the bipulmonary ventilation.

\section{Results}

Thirty-nine patients underwent surgery for lung malformation and one for thymic teratoma during the studied period. The patients were $9 \pm 5.4$ months old and weighed $8 \pm 4.3 \mathrm{~kg}$.

33 patients were affected by cystic adenomatoid malformation, 4 patients by bronchogenic cyst, 2 patient by congenital emphysema, 1 patient by thymic teratoma.
35 patients underwent a thoracoscopy and 5 a thoracotomy because of their low weight. 19 patients underwent a thoracoscopy on the left side and 21 on the right side. The average time for surgery was $139 \mathrm{~min}$.

The bronchial blocker (BB) technique was performed on 7 patients in the right side. In one case intraoperative blocker dislocation occurred and consequently the thoracoscopy was converted into thoracotomy.

The selective intubation (SI) technique was performed on 30 patients. No problem of mobilisation of tube or accidental extubation during the surgery were observed (Table 1).

The patients in the two groups (bronchial blocker vs selective intubation) were

Table 1. Patient characteristics and surgery.

\begin{tabular}{lcc} 
& Min / N. & Max / \% \\
Weigh (kg) & 3 & 13 \\
Age (months) & 0,2 & 23,4 \\
\hline Operative time (min) & 40 & 230 \\
ASA & & \\
ASA 2 & 37 & 92,5 \\
ASA 3 & 3 & 7,5 \\
\hline Lung disease & 33 & 2 \\
Cpam & 82,5 & 5 \\
Bronchogenic cyst & 4 & 1 \\
Cong. Emphysema & 10 & 2,5 \\
Thymic teratoma & & \\
Surgical Technique & 35 & 87,5 \\
Thoracoscopy & 5 & 12,5 \\
Thoracotomy & & \\
Side & 19 & 47,5 \\
$\quad$ Left & 21 & 52,5 \\
Right & & \\
OLV technique & 7 & 17,5 \\
Bronchialblocker & 32 & 80 \\
Selectiveintubation & & \\
\hline
\end{tabular}

$\mathrm{OLV}=$ one lung ventilation.

Table 2. Bronchial blocker and selective intubation.

\begin{tabular}{lccc} 
& Bronchial blocker $(\mathrm{N}=7)$ & Selective intubation (N=25) & P \\
Age (months) & $6,6^{*}(3,3)$ & $10,7(5,2)$ & 0,06 \\
Weight $(\mathrm{kg})$ & $8,1(2,3)$ & $8,6(2,0)$ & 0,53 \\
\hline SpO2 after OLV (\%) & $97(5,1)$ & $97(4,1)$ & 0,79 \\
SpO2 min (\%) & $92(6,0)$ & $94(4,0)$ & 0,3 \\
\hline SpO2 var & $5(4,1)$ & $3.5(2,2)$ & 0,2 \\
etCO2 after OLV & $41(4,9)$ & $43(10)$ & 0,57 \\
\hline etCO2 max & $50(11,5)$ & $51(9,8)$ & 0,92 \\
CO2 var & $10,1(10,3)$ & $8,3(7,9)$ & 0,61 \\
\hline RR & $35(5,4)$ & $37(8,5)$ & 0,6 \\
Vt (mI/kg) & $7,5(1,92)$ & $6,8(2,2)$ & 0,47 \\
\hline Surgery time (min) & $130(14,7)$ & $144(43,7)$ & 0,18 \\
\hline
\end{tabular}

*mean (SD); OLV = one lung ventilation, $\mathrm{RR}$ respiratory rate, $\mathrm{Vt}$ tidal ventilation. compared in terms of baseline characteristics and operative data; The $\mathrm{SpO} 2$ and $\mathrm{ETCO} 2$ registered 5 minutes after OLV (one lung ventilation), have been extracted from anesthesia records as well as minimal/ maximal $\mathrm{SpO} 2$ and ETCO2, respectively; Variations of $\mathrm{SpO} 2$ end ETCO2 were calculated as the difference between the values of $\mathrm{SpO} 2 / \mathrm{ETCO} 25 \mathrm{~min}$ after OLV and the minimal/maximal value of $\mathrm{SpO} 2 / \mathrm{ETCO} 2$ registered during OLV. SpO2 fell on average by 5 points in the BB group and by 3,5 in the SI group. ETCO2 increased by 10 points in the BB group and by 8 in the SI group. There was no significant difference among the groups in the variables considered. Ventilatory parameters (RR, respiratory rate and tidal volume) and duration of surgery 
were also similar among the two groups (Table 2).

26 patients $(65 \%)$ were extubated in the operating room at the end of the operation without adverse events and were transferred to intensive care for postoperative monitoring. 14 patients (35\%) were extubated in intensive care in the following hours. None of the patients underwent blood transfusions.

It's interesting to note that patients extubated in intensive care were all operated before 2016: since that year, in fact, there has been a change in the surgical and anesthesiology team that is more oriented towards early extubation.

The postoperative analgesia was guaranteed with paracetamol $15 \mathrm{mg} / \mathrm{kg}$ every 6 hours and morphine. Upon awakening all patients received a dose of 50$100 \mathrm{mcg} / \mathrm{kg}$ and in intensive care a PCA morphine pump with doses of $25 \mathrm{mcg}$ ( $\max$ $240 \mathrm{mcg} / \mathrm{kg}$ in 4 hours) was set.

The following day all the patients were transferred to the pediatric surgery unit.

\section{Discussion}

There are multiple problems to achieve mono-pulmonary ventilation in newborn and infants. Lateral position problems during anesthesia in mono-pulmonary ventilation are well known. The lower lung is better perfused than the upper one but less ventilated due to gravity. The lower is the only ventilated lung during the surgery. Moreover, the general anesthesia induction reduces the residual functional capacity. All these factors cause an alteration of the ventilation / perfusion ratio and a greater need for oxygen. In newborns and infants, the residual functional capacity is proportionally lower than in adults. Therefore, the shortage of oxygen reserves leads more easily to respiratory failure with lowering of $\mathrm{SpO} 2$ and hypercapnia during monopulmonary ventilation. Potentially, a moderate hypoxia and hypercapnia can be tolerated to avoid forcing the ventilation parameters that can lead to an iatrogenic barotrauma. There are no double-lumen endobronchial tubes for children under $30 \mathrm{~kg}$, so only two choices remain and both require a great experience in pediatric thoracic anesthesia and in the use of fiberscope. Using a bronchial blocker or a surrogate such as a Fogarty catheter which is introduced in the lung bronchus to operate. The blocker is normally placed inside the endotracheal tube but in younger children the presence of the blocker can significantly reduce the internal space of the endotracheal tube. In the latter case the blocker can be introduced laterally to the endotracheal tube before inflating the cuff. The positioning of the blocker is easier on the right side because the right bronchus is better aligned with the trachea. The main drawback of this choice is the possible dislocation of the blocker during surgical maneuvers at the level of the bronchus. If this happens, the lung to be operated is re-ventilated and the surgeon no longer sees the operating field. The only possibility to continue in this case the surgery is to convert the thoracoscopy into thoracotomy or to try to reposition the blocker but this maneuver is extremely difficult especially when the small patient is in a lateral position.

Selectively intubating the bronchus of the contralateral lung. If this maneuver is performed at the level of the right bronchus (for left thoracoscopy) it's necessary to make sure not to close the upper lobar bronchus excluding the upper lobe from ventilation. This can cause a drastic reduction in $\mathrm{SpO} 2$ because the patient has the left lung excluded and a right lung only ventilated through the middle and lower pulmonary lobes. If this maneuver is performed at the level of the left bronchus (for right thoracoscopy) it's imperative to do it under fibroscopic control because positioning the tube in the left bronchus is more difficult than in the right one due to its horizontal trajectory from the trachea. The drawback of this choice is that at the end of the surgery it is necessary to withdraw the tube to have a bi-pulmonary ventilation again and re-expand the operated lung. This maneuver takes place when the patient is still in a lateral position, so an accidental extubation is theoretically possible and intubation in a lateral position may be more difficult than expected. In our experience this problem has not occurred.

\section{Limits of the study}

The study has several limitations: it's a retrospective study, the comparison of the two groups is limited by the small sample size of the "bronchial blocker" group, the blood gas analytical values are not reported because this check is not carried out systematically, it's a single-center study that reflects the practices of a single team.

\section{Conclusions}

The anesthesia for thoracic surgery in newborn and infants is a challenge for anesthetists and require a great experience in pediatric anesthesia and fiberscope use.

The anatomical and physiological peculiarities of children under one year of age expose to hypoxia and hypercarbia problems related to the limited functional residual capacity typical of this age and to the monopulmonary ventilation necessary to allow the surgeon to operate in thoracoscopy.

A permissive moderate hypoxia and hypercarbia must be tolerated to avoid elevated ventilation pressures and consequent iatrogenic barotrauma.

The choice of the best method to carry out mono-pulmonary ventilation depends on selfexperience of the anesthesiologist.

\section{References}

1. Lee EY, Dorkin H, Vargas SO. Congenital Pulmonary Malformations in Pediatric Patients: Review and Update on Etiology, Classification, and Imaging Findings. Radiol Clin North Am 2011;49:921-48.

2. Adewale L. Anatomy and assessment of the pediatric airway. Paediatr Anaesth 2009;19:1-8.

3. Semmelmann A, Kaltofen H, Loop T. Anesthesia of thoracic surgery in children. Pediatr Anesth 2018;1-6

4. Koizumi K, Haraguchi S, Hirata T, et al. Thoracoscopic Surgery in Children. J Nippon Med Sch 2005;72:34-42.

5. Johnson SM, Grace N, Edwards MJ, et al. Thoracoscopic segmentectomy for treatment of congenital lung malformations. J Pediatr Surg 2011;42: 411-4.

6. Adams S, Jobson M, Sangnawakij P, et al. Does thoracoscopy have advantages over open surgery for asymptomatic congenital lung malformations? An analysis of 1626 resections. J Pediatr Surg 2017;52:247-51.

7. Marciniak B. Techniques de ventilation unipulmonaire chez l'enfant. Ann Fr Anesth Reanim 2009;28:678-9.

8. Lammers CR, Hammer GB, Brodsky JB, et al. Failure to Separate and Isolate the Lungs with an Endotracheal Tube Positioned in the Bronchus. Anesth Analg 1997;85:946-7.

9. Marciniak B, Fayoux P, Hébrard A, et al. Fluoroscopic guidance of Arndt endobronchial blocker placement for single-lung ventilation in small children. Acta Anaesthesiol Scand 2008;52:10035.

10. Golianu B, Hammer GB. Pediatric thoracic anesthesia. Curr Opin Anaesthesiol 2005;18:5-11.

11. Guruswamy V, Roberts S, Arnold P, Potter F. Anaesthetic management of a neonate with congenital cyst adenoid malformation. BJA 2005;95:240-2.

12. Letal M, Theam M. Pediatric lung isolation. BJA Education 2016;1-6. 